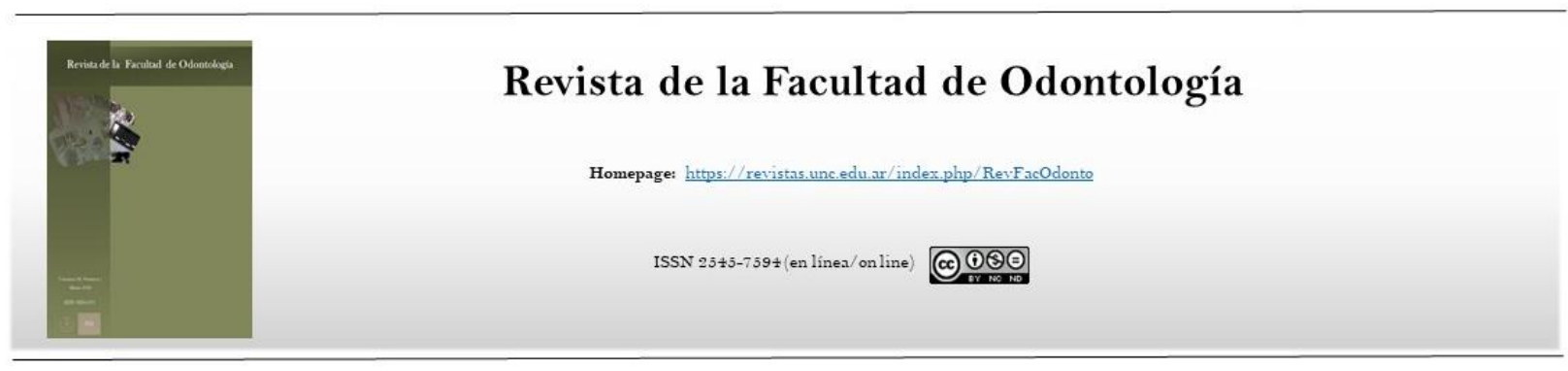

Aplicación de la tecnología Multimedia a la enseñanza de la Farmacología

\title{
Application of Multimedia technology to the teaching of Pharmacology
}

\author{
Virga Carolina ${ }^{1}$, Aramburu Guillermo ${ }^{1}$, De Leonardi Adriana ${ }^{1}$, Ricco Verónica $^{1}$, Escudero Cristina ${ }^{1}$, Hubert Sergio $^{1}$, \\ López Viviana ${ }^{1}$.
}

${ }^{1}$ Universidad Nacional de Córdoba. Facultad de Odontología. Departamento de Patología Bucal. Cátedra de Farmacología y Terapéutica B

\begin{abstract}
The exhibition classes are not very motivating for the students due to the various limitations that include passivity and loss of student attention. A proposal based on an active learning methodology was proposed, seeking that students creatively address the resolution of problematic situations around the contents of the Pain and Inflammation didactic unit, then evaluated through the presentation of videos produced by the students. Material and methods: The experience began with the conduct of a survey among students to learn about previous experience in the use of videos for educational purposes and ultimately assess the initiative implemented. Students were divided into groups and work slogans were established, guiding the teams constituted in the selection of the group of drugs to work. At the end of the Pain and Inflammation Unit, the contents were integrated and the students presented the videos they had produced to address the proposed topics. Results: 20 videos were presented. The valuation of the productions presented was very good from the conceptual, technical, aesthetic point of view and of the contents addressed. All the groups concluded satisfactorily with the elaboration of their recording projects. After the development of the experience, it was concluded that the use of technology becomes an ally to promote the effective learning of Pharmacology, given the benefits obtained from using moving images instead of traditional teaching methods.
\end{abstract}

KEY WORDS: Educational Video; meaningful learning, Multimedia Interactive Learning Styles.

\section{Resumen}

Las clases expositivas son poco motivadoras para los estudiantes por las diversas limitaciones entre las que se encuentran la pasividad y la pérdida de la atención de los alumnos. Se planteó una propuesta basada en una metodología de aprendizaje activo, buscando que los estudiantes abordaran creativamente la resolución de situaciones problemáticas en torno a contenidos de la unidad didáctica de Dolor e Inflamación, luego evaluadas a través de la presentación de videos producidos por los estudiantes. Material: La experiencia se inició con la realización de una encuesta entre los alumnos para conocer la experiencia previa en el uso de videos con fines didácticos y al final valorar la iniciativa implementada. Se dividieron los alumnos en grupos y se establecieron las consignas de trabajo, guiando a los equipos constituidos en la selección del grupo de fármacos a trabajar. Al término de la Unidad de Dolor e Inflamación se realizó la integración de los contenidos y los estudiantes presentaron los videos que habían producido para abordar los temas propuestos. Resultados: Se presentaron 20 videos. La valoración de las producciones presentadas fue muy buena desde el punto de vista conceptual, técnico, estético y de los contenidos abordados. Todos los grupos concluyeron satisfactoriamente con la elaboración de sus proyectos de grabación. Tras el desarrollo de la experiencia se concluyó que el uso de la tecnología se convierte en un aliado para promover el aprendizaje efectivo de la Farmacología, dados los beneficios que se obtienen de utilizar imágenes en movimiento en lugar de los métodos tradicionales de enseñanza.

PALABRAS CLAVE: Video didáctico; aprendizaje significativo, Multimedia Interactivo, Estilos de Aprendizaje

Received 11 March 2020; Received in revised form 25 April 2020; Accepted 7 july 2020 


\section{Introducción}

El término multimedia resulta ya familiar y es frecuente leer cosas sobre las posibilidades que ofrece en los procesos de enseñanza-aprendizaje. Multimedia suele presentarse como el último avance que, propiciado por la evolución y expansión de los medios electrónicos viene a resolver algunos de los problemas que actualmente tiene planteada la enseñanza. Así por ejemplo encontramos que los métodos tradicionales de enseñanza basados principalmente en la transmisión oral por parte del docente, resultan poco atractivos para las nuevas generaciones habituadas a formas de comunicación más ágiles ${ }^{1}$.

El aprendizaje es un proceso comunicativo en el que en los medios didácticos juegan un papel clave en la configuración de las interacciones. Por ello aquellas herramientas, que promueven comunicaciones reales deben destacarse en contraposición a medios que sólo brindan ayudas instructivas sin buscar este tipo de situaciones comunicativas $^{2}$. Precisamente, la multimedia educativa combina las posibilidades de diversos medios de comunicación interconectados y controlados a través de una computadora para el logro de un propósito común: facilitar los procesos de enseñanza-aprendizaje. Estos sistemas tienen ante sí el reto de responder a la impredecibilidad y a la interacción de toda situación comunicativa humana ${ }^{3}$.

La multimedia como herramienta promueve un aprendizaje colaborativo, en el que el resultado de la experiencia colectiva es más que la suma de las partes que intervienen en su construcción, es decir, los aprendizajes que los estudiantes pudieran alcanzar con la experiencia individual. Los resultados se enriquecen al confrontar diversos puntos de vista, diferentes formas de abordaje y diferentes soluciones y propuestas para cada situación ${ }^{4}$.

De acuerdo con estas consideraciones, teóricas antes vertidas desde la Cátedra de Farmacología y Terapéutica B de la Facultad de Odontología de Córdoba, se propuso desarrollar una experiencia de utilización del video como estrategia didáctica para la enseñanza de la Farmacología orientada a la formación de futuros odontólogos. Nuestro interés fue identificar el grado de satisfacción de los estudiantes respecto al uso y la utilidad obtenida al incorporar la tecnología como herramienta didáctica para abordar el contenido de cierre de la unidad didáctica de Dolor e Inflamación.

Los objetivos de este trabajo fueron evaluar la producción y la utilización de recursos multimedia para el aprendizaje de la Farmacología, en la unidad didáctica de dolor e inflamación y valorar la utilidad y efectividad de los recursos multimedia producidos por los estudiantes.

\section{Métodos}

\section{Actividad}

La implementación de la estrategia didáctica se realizó entre abril y junio del año 2018, en la unidad didáctica de Dolor e Inflamación. Se desarrolló con 150 estudiantes que cursaban la asignatura Farmacología y Terapéutica "B" de la Facultad de Odontología Universidad Nacional de Córdoba, Argentina. En esta integración de los temas se les explicó a los alumnos que se llevaría a cabo una experiencia educativa innovadora mediante la producción de un video corto (5 a 7 minutos) donde ellos tuvieran que transmitir a la población datos sobre la prescripción de un grupo de fármacos analgésicos antiinflamatorios a elección dando instrucciones y advertencias con respecto a un tratamiento farmacológico. La actividad desarrollada consistió en el abordaje de las habilidades de comunicación del estudiante con el paciente, en lo que se tuvo especial atención en la prescripción. En cuanto a la parte técnica vinculada en la elaboración del video se consideró la creatividad en la construcción del video.

\section{Instrumentos}

Con el fin de indagar en el dominio que el grupo que el grupo de estudiantes tiene sobre el uso de herramientas tecnológicas se aplicó una encuesta grupal una semana antes de la primera exposición que ellos debían realizar. Para ello se utilizó la ficha de evaluación de aplicaciones multimedia diseñada por Consuelo Belloch Ortí (basada en la Ficha de Evaluación de Pere Marqués, grupo DIM 2003), la cual consta de una caracterización 
general de los contenidos aplicados, requisitos técnicos características técnicas/ estéticas, adecuación como recurso para el aprendizaje o intervención, funcionalidad, observaciones (aspectos positivos y negativos) y valoración global.

Este cuestionario se volvió a aplicar el día de la presentación de los videos. El objetivo de esta segunda aplicación de la encuesta se vinculó con indagar si los estudiantes mantenían su misma posición manifestada antes de participar en la creación de sus propios videos. Luego de presentados los videos, los docentes a cargo de la experiencia promovieron un espacio para la discusión y análisis tanto de los contenidos abordados por cada grupo en su video, como de la experiencia vivida a la hora de elaborar tan enriquecedoras producciones.

\section{La propuesta didáctica}

El abordaje de la unidad se efectuó a través de la resolución de casos clínicos reales, que promovieron la búsqueda de literatura científica y la realización de prescripciones odontológica. Finalizado este abordaje, la clase se organizó en grupos de cuatro o cinco estudiantes con la finalidad de producir un video que mostrara la resolución del caso. Para ello, se posibilitó a los estudiantes, durante el mes de junio, que trabajaran en el desarrollo del video durante sesenta minutos dentro del horario de trabajo teórico-práctico regular. En este tiempo se compartieron experiencias y se aclararon dudas no sólo sobre la técnica, sino también sobre el contenido que debían incluir en sus producciones. Todos los estudiantes del curso poseían un dispositivo para la grabación de imágenes.

Al término de la Unidad de Dolor e inflamación se realizó la integración de los temas, una habilidad o capacidad intelectual que se adquiere y se desarrolla mediante ejercicios y actividades de aprendizaje diseñadas para tal fin. En ese espacio los estudiantes presentaron en los mismos grupos de trabajo el video que habían producido para abordar los temas propuestos.

\section{Evaluación}

Para evaluar las producciones realizadas por los estudiantes se utilizó la ficha de evaluación de aplicaciones multimedia, Se distribuyeron 20 fichas, correspondientes a los 20 videos producidos.

Este instrumento consideró: a) los requisitos técnicos de la aplicación, b) la calidad de la misma y c) diseño y la estética.

Los requisitos técnicos deben tenerse en cuenta para conocer su posible uso en una computadora con unas prestaciones concretas. Para estos materiales se tendrán en cuenta múltiples aspectos técnicos, expresivos, estéticos, pedagógicos y funcionales, considerando en todo momento que se trata de un producto audiovisual de tipo interactivos multimedia. Por otro lado, estas características técnicas de la aplicación determinaran de algún modo la calidad técnica de la aplicación.

El $90 \%$ de ellos era dueño de un teléfono inteligente, el restante $10 \%$ no tenía celular con cámara, pero disponía de cámara digital, lo cual para los fines del estudio era satisfactorio.

La calidad del entorno visual resulta de gran relevancia en las aplicaciones multimedia interactivas orientadas al aprendizaje, dado que el objetivo de los materiales multimedia aplicados al terreno educativo no es deslumbrar, asombrar o divertir, sino enseñar e instruir, será necesario tener presentes los siguientes principios básicos: simplicidad, coherencia, claridad, adaptabilidad, junto con consideraciones estéticas como son el equilibrio, la armonía y la unidad, la utilización del espacio en blanco, y el tiempo.

\section{Resultados}

Los datos recolectados a través de la ficha de evaluación de aplicaciones multimedia arrojaron que el $70 \%$ de las producciones realizadas presenta muy buena calidad. La evaluación de las características técnicas, calidad de componentes Multimedia, fluidez, velocidad de procesamiento y presentación de la información, el 70\% presenta muy buena calidad. En cuanto a los aspectos estéticos el dato más importante es la alta calidad del entorno visual que ronda el $90 \%$. (datos no mostrados)

En cuanto a la adecuación como recurso para el aprendizaje o intervención la variable lenguaje adecuado para el usuario obtuvo un el $72,22 \%$ de 
respuestas presenta un lenguaje claro, coherente y adecuado para los receptores. Fig. 1.

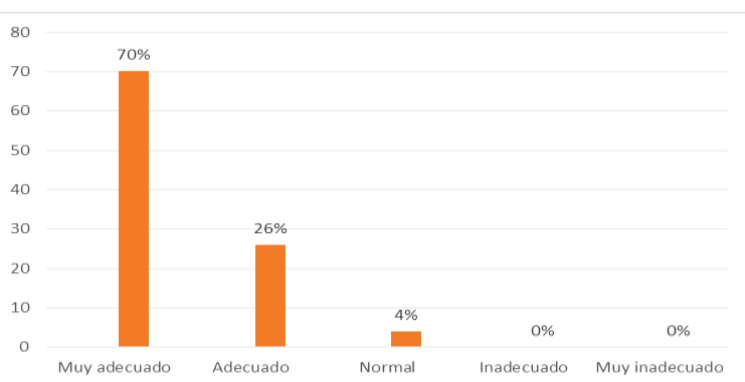

Figura 1. Características técnicas y calidad de componentes multimedia

La Organización y adecuación de los contenidos mostró un 83\% de efectividad. Fig. 2.

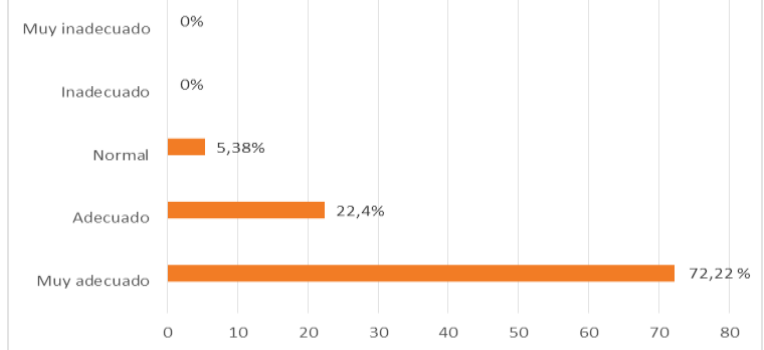

Figura 2. Vocabulario de la TIC con respecto al usuario receptor

El análisis de la adecuación de los componentes multimedia revela un $77 \%$ de efectividad para el diagrama de textos, imágenes y sonidos. Fig. 3.

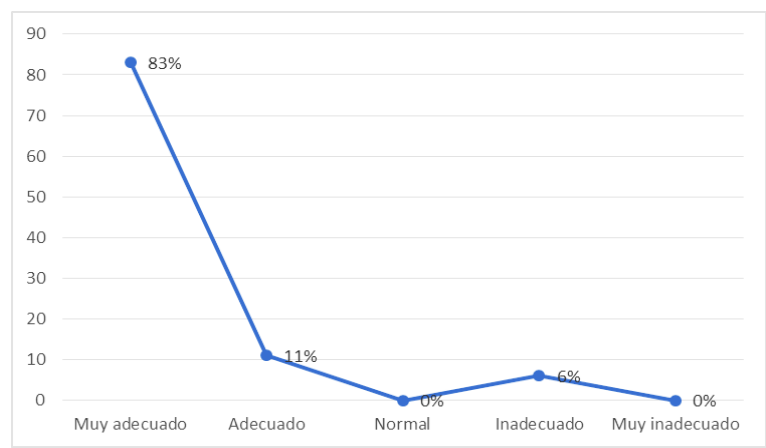

Figura 3. Adecuación de los contenidos

Los ritmos de aprendizaje reflejados en esta actividad de aprendizaje mostraron un $80 \%$ en la adecuación a las consignas presentadas, Fig. 4.

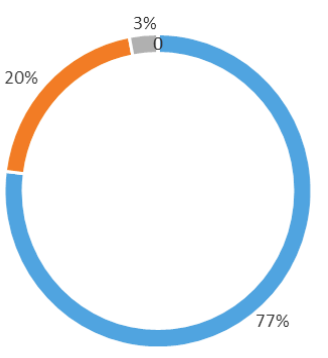

Figura 4. Adecuación de componentes multimedia

El grado de adecuación como recurso para el aprendizaje en cuanto a objetivos propuestos, contenidos, vocabulario, componentes multimedia y estrategias didácticas mostró un $80 \%$ como Muy Adecuado mientras que el $20 \%$ restante fue adecuado (Fig. 5).

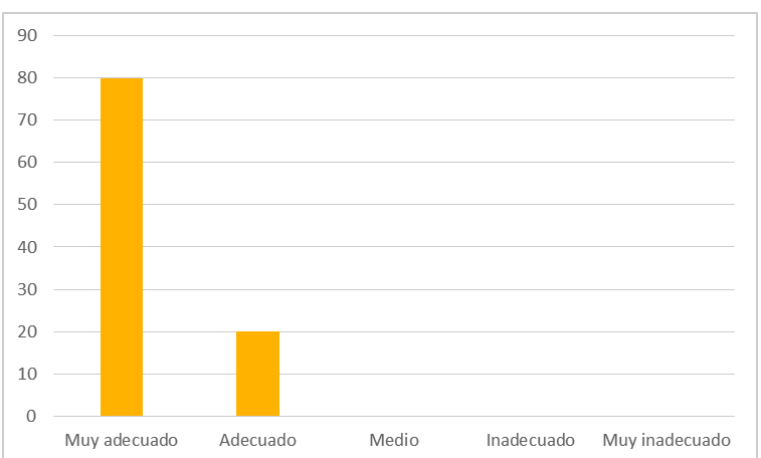

Figura 5. Adecuación como recurso para el aprendizaje e intervención

La valoración general de los 20 videos presentados fue muy buena desde el punto de vista técnico, estéticos y la relación enseñanza aprendizaje aplicado a estas nuevas tecnologías.

\section{Discusión y Conclusión}

Con esta propuesta se quiso plantear una actividad innovadora respecto de los patrones tradicionales a la hora de abordar contenidos de dolor e inflamación en la enseñanza de la Farmacología. En general, se puede considerar que la creación de videos didácticos, por parte de los estudiantes como estrategia a desarrollar en una clase fue bien vista por todos los jóvenes. El logro principal fue que el abordaje del contenido resultó más 
atractivo y despertó el interés en la temática motivando a los alumnos a un abordaje más profundo y comprometido.

$\mathrm{Si}$ bien, los participantes, en su mayoría, reconocieron que nunca habían trabajado en la producción y edición de videos, esta situación generó un poco de incertidumbre al inicio porque no poseían los conocimientos suficientes para trabajar con este tipo de programas, sin embargo, dado que la idea les resultó atractiva lograron superar los obstáculos derivados del desconocimiento de la técnica de edición de videos. Todos los grupos concluyeron satisfactoriamente con la elaboración de sus proyectos de grabación, pese a que la totalidad de los estudiantes aseguró que les hubiese gustado trabajar mayor tiempo para realizar sus videos.

Por medio de la producción de sus propios videos, los estudiantes reforzaron la autoconfianza y autodeterminación en cuanto a su desempeño académico. Se fortaleció el desarrollo de un proceso de aprendizaje consciente, en el cual, los cursantes de farmacología asumieron un rol responsable hacia la construcción de su propio conocimiento.

La valoración general de los videos presentados y realizados a través de esta tecnología, fue muy buena desde el punto de vista conceptual, técnico y estético. Además, el método fue muy efectivo tanto desde la óptica de la enseñanza como del aprendizaje. Es así, que creemos que este tipo de tecnologías multimedia orientadas a la enseñanza de la Farmacología en el grado facilita no solo la comprensión de los contenidos de la asignatura, sino que también, se constituye en una herramienta valiosa para el desarrollo de procesos de aprendizaje más comprometidos y significativos por parte de los estudiantes.

\section{Agradecimientos}

A la Mag. Adriana Tessio a quien nos gustaría expresar nuestro agradecimiento profundo por la supervisión de este trabajo, además de agradecer su tiempo, su paciencia y dedicación para mejorar estas líneas.

Los autores declaran que no existen conflictos potenciales de interés con respecto a la autoría y / o publicación de este artículo.

The authors declare no potential conflicts of interest with respect to the authorship and/or publication of this article

\section{Referencias}

1. Boude Figueredo O, Medina Rivilla A., Desarrollo de competencias a través de un ambiente de aprendizaje mediado por TIC en educación superior. Educ Med Super [Internet]. 2011; 25(3). Disponibleen:http://scieloprueba.sld.cu/scielo.php?scri pt=sci_arttext\&pid=S086421412011000300007\&lng=es

2. Cuenca Doimeadios E, Reyes Hernández D, Ellis Yards ML, Navarro Hernández M, Alvelo Pérez D., Recursos de aprendizaje en la asignatura de Psiquiatría para la formación del Médico Integral Comunitario. EducMedSuper [Internet]. 2013; 27(1). Disponible en: http://scieloprueba.sld.cu/scielo.php?script=sci_arttext \&pid=S0864- 21412013000100005\&lng=es

3. García Garcés H, Navarro Aguirre L, López Pérez M, Rodríguez Orizondo MF., Las Tecnologías de la Información y la Comunicación en la salud y la educación médica. EDUMECENTRO [Internet]. 2014; 6(1). Disponible en: http://www.revedumecentro.sld.cu/index.php/edumc/ar ticle/view/373/570

4. Puig Rosell W, González Hourruitiner A., Criterios de clasificación y selección de los medios de enseñanza. Educ Med Super [Internet].http://www.revedumecentro.sld.cu 90 2014] 2012; 26(2). Disponible en: http://scieloprueba.sld.cu/scielo.php?script=sci_arttext \&pid=S0864- 21412012000200015\&lng=es

Corresponding to /correspondencia a: Prof. Dra Carolina Virga Cátedra B de Farmacología y Terapéutica Departamento de Patología Bucal. Facultad de Odontología Universidad Nacional de Córdoba. Haya de la Torre s/ $n$ ciudad universitaria Córdoba Argentina. Correo electrónicole-mail: maria.virga@unc.edu.ar 